\title{
DYNAMICS OF SPLIT POLYNOMIAL MAPS: UNIFORM BOUNDS FOR PERIODS AND APPLICATIONS
}

\author{
D. GHIOCA AND K. D. NGUYEN
}

\begin{abstract}
Let $K$ be an algebraically closed field of characteristic 0 . Following Medvedev-Scanlon, a polynomial of degree $\delta \geq 2$ is said to be disintegrated if it is not linearly conjugate to $x^{\delta}$ or $\pm T_{\delta}(x)$ where $T_{\delta}(x)$ is the Chebyshev polynomial of degree $\delta$. Let $d$ and $n$ be integers greater than 1 , we prove that there exists an effectively computable constant $c(d, n)$ depending only on $d$ and $n$ such that the following holds. Let $f_{1}, \ldots, f_{n} \in K[x]$ be disintegrated polynomials of degree at most $d$ and let $\varphi=f_{1} \times \cdots \times f_{n}$ be the induced coordinate-wise self-map of $\mathbb{A}_{K}^{n}$. Then the period of every irreducible $\varphi$-periodic subvariety of $\mathbb{A}_{K}^{n}$ with non-constant projection to each factor $\mathbb{A}_{K}^{1}$ is at most $c(d, n)$. As an immediate application, we prove an instance of the dynamical Mordell-Lang problem following recent work of Xie. The main technical ingredients are Medvedev-Scanlon classification of invariant subvarieties together with classical and more recent results in Ritt's theory of polynomial decomposition.
\end{abstract}

\section{INTRODUCTION}

Throughout this paper, let $\mathbb{N}$ denote the set of positive integers, $\mathbb{N}_{0}:=$ $\mathbb{N} \cup\{0\}$, and let $K$ be an algebraically closed field of characteristic 0 . For a map $\mu$ from a set to itself and for $m \in \mathbb{N}$, we let $\mu^{\circ m}$ denote the $m$-fold iterate $\mu \circ \ldots \circ \mu$. For every polynomial $f(x) \in K[x]$, the notations $f^{\circ m}, f^{\circ m}(x)$, and $f(x)^{\circ m}$ have the same meaning which is the $m$-fold iterate $f \circ \ldots \circ f$.

Let $\delta \geq 2$ be an integer, the Chebyshev polynomial $T_{\delta}(x) \in K[x]$ is the polynomial of degree $\delta$ satisfying $T_{\delta}\left(x+\frac{1}{x}\right)=x^{\delta}+\frac{1}{x^{\delta}}$. Following the terminology in Medvedev-Scanlon [MS14], we say that a polynomial $f(x) \in$ $K[x]$ is disintegrated if it has degree $\delta \geq 2$ and it is not linearly conjugate to $x^{\delta}$ or $\pm T_{\delta}(x)$. Let $n \in \mathbb{N}$ and let $f_{1}, \ldots, f_{n} \in K[x]$ be polynomials of degree at least 2 . Let $\varphi=f_{1} \times \cdots \times f_{n}$ be the induced coordinate-wise self-map of $\mathbb{A}_{K}^{n}$. An irreducible subvariety $V$ of $\mathbb{A}_{K}^{n}$ is said to be $\varphi$-periodic if there exists $N \in \mathbb{N}$ satisfying $\varphi^{\circ N}(V)=V$; the smallest such $N$ is called the period (or more precisely $\varphi$-period) of $V$. According to [MS14, Theorem 2.30], to study the algebraic dynamics of $\varphi$, it suffices to study two cases: the case when none of the $f_{i}$ 's are disintegrated which reduces to the geometry

2010 Mathematics Subject Classification. Primary: 37P05. Secondary: 14G99.

Key words and phrases. split polynomial maps, periods, polynomial semiconjugacy, Medvedev-Scanlon classification. 
of $\mathbb{G}_{\mathrm{m}}^{n}$ (see, for instance, [BG06, Chapter 3]) and the case when all the $f_{i}$ 's are disintegrated. In the first case, we note that the period under the multiplication-by- $d$ map of a subvariety of $\mathbb{G}_{\mathrm{m}}^{2}$ can be as large as possible. For instance, the torsion translate defined by $x=\zeta y$ where $\zeta$ is a primitive $\left(d^{N}-1\right)$-th root of unity has period $N$ under the map $(x, y) \mapsto\left(x^{d}, y^{d}\right)$. On the other hand, we obtain the following result in the latter case:

Theorem 1.1. Let $n \geq 1$ and $d \geq 2$ be integers, then there is a an effectively computable constant $c(d, n)$ depending only on $d$ and $n$ such that the following holds. Let $f_{1}, \ldots, f_{n} \in K[x]$ be disintegrated polynomials of degree at most $d$. Let $\varphi:=f_{1} \times \cdots \times f_{n}$ be the induced coordinate-wise self-map on $\mathbb{A}_{K}^{n}$. Let $V$ be an irreducible $\varphi$-periodic subvariety of $\mathbb{A}_{K}^{n}$ such that the projection from $V$ to each factor $\mathbb{A}_{K}^{1}$ is non-constant. Then the period of $V$ is at most $c(d, n)$.

In Theorem 1.1, the condition that the projection to each factor $\mathbb{A}_{K}^{1}$ is non-constant is necessary. For instance, let $\zeta_{1}$ be periodic under $f_{1}$, then the $\varphi$-period of $\left\{\zeta_{1}\right\} \times \mathbb{A}_{K}^{n-1}$ is the $f_{1}$-period of $\zeta_{1}$ which can be arbitrarily large. Following the proof (see Section 6 ), the constant $c(d, 2)$ can be taken to be $\frac{d^{2 d^{4}}}{2}$ and for $n \geq 3, c(d, n)$ can be defined recursively in an explicit way. It is conceivable that such constants are not best possible; the goal of our theorem is to show that they depend only on $d$ and $n$, and they are independent on the actual coefficients of the polynomials $f_{i}$. The proof of Theorem 1.1 uses the classification of $\varphi$-period subvarieties by Medvedev-Scanlon [MS14] and a uniform bound involving semiconjugacy relation among disintegrated polynomials that might be of independent interest (see Section 2, in particular Theorem 2.3).

As an application of our Theorem 1.1, we prove the following instance (see Theorem 1.2) of the Dynamical Mordell-Lang Conjecture following recent work of Junyi Xie [Xie]. For a survey on this conjecture, we refer the readers to the upcoming book of Jason Bell, Thomas Tucker, and the first author [BGT]. By an arithmetic progression, we mean a set of the form $\{a+b k$ : $\left.k \in \mathbb{N}_{0}\right\}$ for some $a, b \in \mathbb{N}_{0}$. Note that this definition also includes singletons (when $b=0$ ). We have the following:

Theorem 1.2. Let $F_{1}, \ldots, F_{n} \in K[x]$ and let $\Phi=F_{1} \times \cdots \times F_{n}$ be the induced coordinate-wise self-map of $\mathbb{A}_{K}^{n}$. Let $C \subset \mathbb{A}_{K}^{n}$ be a curve, and let $\alpha \in \mathbb{A}^{n}(K)$. Then the set $\left\{m \in \mathbb{N}: \Phi^{\circ m}(\alpha) \in C\right\}$ is a finite union of arithmetic progressions.

Theorem 1.2 when $K=\overline{\mathbb{Q}}$ was obtained by Xie [Xie, Theorem 0.3 ]; we note that $[\mathrm{Xie}]$ is a veritable tour de force - an almost 100 pages long paper which proves the Dynamical Mordell-Lang Conjecture for plane curves. Theorem 1.2 removes the condition $K=\overline{\mathbb{Q}}$ and it is proven by combining Xie's result together with standard specialization arguments using the uniform bound from Theorem 1.1. During the preparation of this paper, we 
learned from Xie that after some further steps and a more careful analysis of the arguments in [Xie], he is able to extend his results over any field. However, Xie's extension of his [Xie, Theorem 0.3] from $K=\overline{\mathbb{Q}}$ to an arbitrary field $K$ uses completely different arguments than the ones we employ, since he does not prove a uniform bound for the period of a variety as in our Theorem 1.1. Instead, Xie told us that he needs to re-write his proof from [Xie] in a more general setup since his arguments do not easily extend from $\overline{\mathbb{Q}}$ to an arbitrary field using specialization theorems. On the other hand, the main result of our paper (Theorem 1.1) provides a very direct route to proving Theorem 1.2 using specialization techniques.

The organization of this paper is as follows. In the next section, we present a theorem involving semiconjugate polynomials (see Theorem 2.3). In order to prove this theorem, we need some classical and new results in Ritt's theory of polynomial decomposition as proven in the seminal paper [ZM]. Then we introduce the Medvedev-Scanlon classification of periodic subvarieties under split polynomial maps [MS14] and give the proof of Theorem 1.1. The proof of Theorem 1.2 is given in the last section.

Acknowledgments. We are grateful to Alice Medvedev, Fedor Pakovich, Thomas Scanlon, and Junyi Xie for useful discussions. The first author is partially supported by NSERC and the second author is partially supported by a UBC-PIMS postdoctoral fellowship.

\section{Uniform Bounds for Semiconjugate Polynomials}

Definition 2.1. Let $f, \eta \in K[x]$ be polynomials of degree at least 2. The polynomial $\eta$ is said to be semiconjugate to $f$ if there is a non-constant polynomial $p(x) \in K[x]$ such that $f \circ p=p \circ \eta$.

For recent results on semiconjugate polynomials, we refer the readers to Pakovich's paper [Pak]. It is not difficult to prove that if $\eta$ is semiconjugate to $f$ then $f$ is disintegrated if and only if $\eta$ is disintegrated (see, for instance, [Pak, Theorem 4.4]). We define the relation $\approx$ in the set of disintegrated polynomials:

Definition 2.2. For any disintegrated polynomials $f$ and $g$ in $K[x]$, we write $f \approx g$ if there exist $N \in \mathbb{N}$ such that there is a polynomial $\eta$ that is semiconjugate to both $f^{\circ N}$ and $g^{\circ N}$.

According to [MS14, Corollary 2.35], $f \approx g$ if and only if the self-map $(x, y) \mapsto(f(x), g(y))$ of $\mathbb{A}^{2}$ admits a periodic curve $C$ having non-constant projection to each factor $\mathbb{A}^{1}$. Then it is not difficult to show that $\approx$ is an equivalence relation in the set of disintegrated polynomials (see [GN, Section 7.1]). As explained in [GN, Section 7.1], if $f_{1}, \ldots, f_{m}$ are in the same equivalence class under $\approx$, then there is $N \in \mathbb{N}$ such that there exists a common polynomial $\eta$ that is semiconjugate to each of the polynomial $f_{i}^{\circ N}$. Besides the Medvedev-Scanlon classification, the key result to the proof of Theorem 1.1 is the following: 
Theorem 2.3. Let $d \geq 2$ be an integer. Let $f$ and $g$ be disintegrated polynomials in $K[x]$ of degree at most $d$ satisfying $f \approx g$. Then there exists $N \leq 2 d^{4}$ and a common polynomial $\eta \in K[x]$ that is semiconjugate to both $f^{\circ N}$ and $g^{\circ N}$.

By a simple induction argument, we have the following extension of Theorem 2.3:

Corollary 2.4. Let $d$ and $n$ be integers at least 2, then there exists an effectively computable constant $c_{1}(d, n)$ depending only on $d$ and $n$ such that the following holds. Let $f_{1}, \ldots, f_{n}$ be disintegrated polynomials of degree at most $d$ belonging to the same equivalence class under $\approx$. Then there exist a positive integer $N \leq c_{1}(d, n)$ and a polynomial $\theta \in K[x]$ that is semiconjugate to $f_{i}^{\circ N}$ for $1 \leq i \leq n$.

The proof of Theorem 2.3 uses classical and more recent results in Ritt's theory of polynomial decomposition as presented in the paper by Müller and Zieve [ZM] and Inou [Ino11]. For another remarkable uniform bound in polynomial decomposition, we refer the readers to [ZM, Theorem 1.4] which also has applications to the Dynamical Mordell-Lang Conjecture [GTZ12].

We now introduce an interesting result of Pakovich and a consequence of his result and of Corollary 2.4. The rest of this section is not needed for the proof of Theorem 2.3. We have [Pak, Theorem 1.4]:

Theorem 2.5 (Pakovich). Let $n \geq 2$ be an integer, then there exists a constant $c_{3}(n)$ depending only on $n$ such that the following holds. Let $B \in$ $K[x]$ be a disintegrated polynomial of degree $n$, let $\mathcal{F}(B)$ denote the set of polynomials $P(x) \in K[x]$ such that $P \circ S=S \circ B$ for some non-constant $S(x) \in K[x]$ (i.e. $B$ is semiconjugate to $P$ ). Then there is a such a pair $(P, S)$ with $\operatorname{deg}(S) \leq c_{3}(n)$ that is universal in the following sense. For every $Q(x) \in \mathcal{F}(B)$, there exist non-constant $S_{1}(x), S_{2}(x) \in K[x]$ such that: $S=S_{2} \circ S_{1}, P \circ S_{2}=S_{2} \circ Q$, and $Q \circ S_{1}=S_{1} \circ B$.

By combining Corollary 2.4 and Theorem 2.5, we have the following:

Corollary 2.6. Let $d$ and $n$ be integers at least 2, then there exists a constant $c_{4}(d, n)$ depending only on $d$ and $n$ such that the following holds. Let $f_{1}, \ldots, f_{n}$ be disintegrated polynomials of degree at most $d$ belonging to the same equivalence class under $\approx$. Then there exist a positive integer $N \leq c_{1}(d, n)$, polynomials $\theta$ and $P$ of degree $d^{N}$, a non-constant polynomial $S$, and non-constant polynomials $S_{i, 2}, S_{i, 1}$ of degree at most $c_{4}(d, n)$ for $1 \leq i \leq n$ such that: $f_{i}^{\circ N} \circ S_{i, 1}=S_{i, 1} \circ \theta, P \circ S_{i, 2}=S_{i, 2} \circ f_{i}^{\circ N}$, and $S=S_{i, 2} \circ S_{i, 1}$ for $1 \leq i \leq n$.

Proof. By Corollary 2.4, there exist a positive integer $N \leq c_{1}(d, n)$ and a polynomial $\theta$ such that $f_{i}^{\circ N} \in \mathcal{F}(\theta)$ for $1 \leq i \leq n$. The desired result now follows from Theorem 2.5. 


\section{Results in RitT's theory of polynomial DeCOMPosition}

The following result by Engstrom [Eng41] will be used repeatedly in this paper:

Lemma 3.1. If $a, b, c, d \in K[x] \backslash K$ satisfy $a \circ b=c \circ d$ then there exist $\hat{a}, \hat{b}, \hat{c}, \hat{d}, g, h \in \mathbb{C}[x]$ such that:

- $g \circ \hat{a}=a, g \circ \hat{c}=c, \operatorname{deg}(g)=\operatorname{gcd}(\operatorname{deg}(a), \operatorname{deg}(c))$.

- $\hat{b} \circ h=b, \hat{d} \circ h=d, \operatorname{deg}(h)=\operatorname{gcd}(\operatorname{deg}(b), \operatorname{deg}(d))$.

- $\hat{a} \circ \hat{b}=\hat{c} \circ \hat{d}$.

Consequently, if $\operatorname{deg}(a)=\operatorname{deg}(c)$ then there is a linear polynomial $\ell$ such that $a=c \circ \ell$ and $b=\ell^{-1} \circ d$.

Ritt's second theorem [Rit22] (also see [Tor88] or [ZM, Appendix]) then allows us to study the equation $\hat{a} \circ \hat{b}=\hat{c} \circ \hat{d}$ under the condition $\operatorname{gcd}(\operatorname{deg}(\hat{a}), \operatorname{deg}(\hat{c}))=$ $\operatorname{gcd}(\operatorname{deg}(\hat{b}), \operatorname{deg}(\hat{d}))=1$. For a version of Ritt's theorem including the case of positive characteristic, we refer the readers to Schinzel's book [Sch00] following an earlier paper of Zannier [Zan93]. We have the following immediate consequence of Ritt's theorem for semiconjugacy functional equations:

Lemma 3.2. Let $f$ be a disintegrated polynomial of degree $\delta$. Let $p$ and $\eta$ be non-constant polynomials such that $f \circ p=p \circ \eta$ and $\operatorname{gcd}(\delta, \operatorname{deg}(p))=1$. Then there exist linear polynomials $\ell_{1}, \ell_{2}, \ell_{3}$, positive integers $b, c$, and $a$ non-constant polynomial $P$ such that the following hold:

- $P(0) \neq 0$ and $c \equiv b$ modulo $\delta$.

- $\ell_{1} \circ f \circ \ell_{1}^{-1}=x^{c} P(x)^{b}$.

- $\ell_{1} \circ p \circ \ell_{2}^{-1}=x^{b}$.

- $\ell_{2} \circ \eta \circ \ell_{2}^{-1}=x^{c} P\left(x^{b}\right)$.

Proof. See [Ino11, Appendix A].

Following [ZM, Section 3], we have the following definitions:

Definition 3.3. Two non-constant polynomials $A$ and $B$ are said to be equivalent if there are linear polynomials $L_{1}$ and $L_{2}$ such that $L_{2} \circ A \circ L_{1}=B$. Write $\delta=\operatorname{deg}(A)$. The polynomial $A$ is said to be cyclic if $A$ is equivalent to $x^{\delta}$ and $\delta \geq 2$. The polynomial $A$ is said to be dihedral if $A$ is equivalent to $T_{\delta}(x)$ and $\delta \geq 3$.

Definition 3.4. Let $A$ be a non-constant polynomial. The group $\Gamma(A)$ is defined to be the group (under composition) of linear polynomials $\ell$ such that $A \circ \ell=L \circ A$ for some linear polynomial $L$.

We have the following lemmas:

Lemma 3.5. Let $F$ be a disintegrated polynomial. Then $F^{\circ 2}$ is not cyclic; moreover $F^{\circ 2}$ is not dihedral if $\operatorname{deg}(F) \geq 3$. Consequently, $f^{\circ 4}$ is neither cyclic nor dihedral for every disintegrated polynomial $f$. 
Proof. Write $\delta=\operatorname{deg}(F) \geq 2$. First assume that $F^{\circ 2}$ is cyclic, hence $F^{\circ 2}=$ $L_{1} \circ x^{\delta^{2}} \circ L_{2}$ for some linear polynomials $L_{1}$ and $L_{2}$. By Lemma 3.1, there is a linear polynomial $L$ such that:

$$
F=L_{1} \circ x^{\delta} \circ L=L^{-1} \circ x^{\delta} \circ L_{2} .
$$

Hence $L \circ L_{1} \circ x^{\delta}=x^{\delta} \circ L_{2} \circ L^{-1}$. By comparing the coefficients of $x^{\delta-1}$, we have that $L_{2} \circ L^{-1}=\gamma x$, hence $L_{2}=\gamma L$. This gives $F=L^{-1} \circ \gamma^{\delta} x^{\delta} \circ L$ which is linearly conjugate to $\gamma^{\delta} x^{\delta}$, hence to $x^{\delta}$, contradiction.

Now assume that $\delta \geq 3$ and $F^{\circ 2}$ is dihedral. By similar arguments, we have linear polynomials $L_{1}, L_{2}, L$ such that:

$$
F=L_{1} \circ T_{\delta} \circ L=L^{-1} \circ T_{\delta} \circ L_{2} .
$$

Therefore $L \circ L_{1} \circ T_{\delta}=T_{\delta} \circ L_{2} \circ L^{-1}$ which yields $L_{2} \circ L^{-1}= \pm x$; this fact follows using the fact that the coefficient of $x^{\delta-1}$ in $T_{\delta}$ is 0 while the coefficient of $x^{\delta-2}$ in $T_{\delta}$ is nonzero. Thus $L_{2}= \pm L$, and so, we have $F=$ $L^{-1} \circ T_{\delta}( \pm x) \circ L$ which is linearly conjugate to $\pm T_{\delta}(x)$, contradiction.

The last assertion follows from applying the previous assertions for $F=$ $f^{\circ 2}$ (note that $f^{\circ 2}$ is also disintegrated).

Lemma 3.6. Let $A$ be a polynomial of degree $\delta \geq 2$. The following hold:

(a) Let $L_{1}$ and $L_{2}$ be linear polynomials. Then the map $\ell \mapsto L_{2}^{-1} \circ \ell \circ L_{2}$ is an isomorphism from $\Gamma(A)$ to $\Gamma\left(L_{1} \circ A \circ L_{2}\right)$.

(b) For every positive integer $n, \Gamma\left(f^{\circ n}\right)$ is a subgroup of $\Gamma$.

(c) $\Gamma(A)$ is infinite if and only if $A$ is cyclic.

(d) Assume that $\Gamma(A)$ is finite of order $n$. Then $A$ is equivalent to a polynomial of the form $x^{s} P\left(x^{n}\right)$ where $P$ is not a polynomial in $x^{j}$ for any $j \geq 2$. Consequently, $\Gamma$ is cyclic of order $n \leq \delta / 2$.

(e) If $A$ is equivalent to a polynomial of the form $x^{s} P\left(x^{n}\right)$ with $s>0$, $n \geq 2$ and non-constant $P(x) \in K[x] \backslash x K[x]$, then $A$ is not cyclic.

Proof. Part (a) is straightforward. For parts (c) and (d), see [ZM, Lemma 3.17]. For part (b), let $\ell \in \Gamma\left(f^{\circ n}\right)$, therefore $f^{\circ n} \circ \ell=L \circ f^{\circ n}$ for some linear polynomial $L$. Then Lemma 3.1 gives that $f \circ \ell=L_{1} \circ f$ for some linear $L_{1}$. This gives $\ell \in \Gamma(f)$ and proves part (b).

For part (e), assume that there are linear polynomials $L_{1}$ and $L_{2}$ such that $x^{s} P\left(x^{n}\right) \circ L_{1}=L_{2}\left(x^{\delta}\right)$. By comparing the coefficients of $x^{\delta-1}$ and the constant coefficients, we have that $L_{1}(x)=a x$ and $L_{2}(x)=b x$. Since $P(x) \notin x K[x]$, we get a contradiction.

Lemma 3.7. Let $f$ and $g$ be polynomials of degree $\delta \geq 2$ both of which are not cyclic. Assume there exist a linear polynomial $L$ and a positive integer $n \geq \frac{\delta+1}{2}$ such that $f^{\circ n}=L \circ g^{\circ n}$. Then there exist a linear polynomial $\ell$ and a positive integer $N \leq \frac{\delta}{2}$ such that $f^{\circ N}=\left(\ell \circ g \circ \ell^{-1}\right)^{\circ N}$.

Proof. Applying Lemma 3.1 repeatedly, there exist linear polynomials $L_{0}(x):=$ $x, L_{1}, \ldots, L_{n-1}, L_{n}:=L$ such that:

$f=L_{1} \circ g=L_{2} \circ g \circ L_{1}^{-1}=L_{3} \circ g \circ L_{2}^{-1}=\ldots=L_{n-1} \circ g \circ L_{n-2}^{-1}=L \circ g \circ L_{n-1}^{-1}$. 
From $L_{1} \circ g=L_{i+1} \circ g \circ L_{i}^{-1}$, we have that $L_{i} \in \Gamma(g)$ for every $0 \leq i \leq n-1$. Since $|\Gamma(g)| \leq \delta / 2<n$, there exist $0 \leq i<j \leq \delta / 2$ such that $L_{i}=L_{j}$. We now have:

$$
f^{\circ(j-i)}=\left(L_{j} \circ g \circ L_{j-1}^{-1}\right) \circ \ldots \circ\left(L_{i+1} \circ g \circ L_{i}^{-1}\right)=L_{j} \circ g^{\circ(j-i)} \circ L_{i}^{-1} .
$$

Since $L_{j}=L_{i}$, this finishes the proof.

Lemma 3.8. Let $A, B, P \in K[x] \backslash K$ and let $s$ and $n$ be coprime positive integers. If $A \circ B=x^{s} P(x)^{n}$ then $a=x^{j} P_{1}(x)^{n} \circ \ell$ and $b=\ell^{-1} \circ x^{k} P_{2}(x)^{n}$ for some $j, k>0$ satisfying $\operatorname{gcd}(j, n)=\operatorname{gcd}(k, n)=1$, and some $P_{1}, P_{2}, \ell \in K[x]$ with $\ell$ linear.

Proof. This is proved in [ZM, Lemma 3.11].

Lemma 3.9. Let $s>0$ and $n \geq 2$ be coprime integers. Let $P \in K[x] \backslash x K[x]$, and suppose that $f(x):=x^{s} P(x)^{n}$ is neither cyclic nor dihedral. Then for any linear polynomials $\ell_{1}, \ell_{2}$ satisfying $\ell_{1} \circ f \circ \ell_{2}=x^{\tilde{s}} P_{1}(x)^{\tilde{n}}$ with coprime integers $\tilde{s}>0, \tilde{n} \geq 2$, and polynomial $P_{1}(x) \in K[x] \backslash x K[x]$, we have that $\ell_{1}(x)=\gamma x$.

Proof. This is proved in [ZM, Lemma 3.21].

We have the following:

Lemma 3.10. Let $f$ be a disintegrated polynomial and let $N \geq 4$. Assume there are coprime integers $s>0, n>1$, and non-constant polynomial $P(x) \in K[x] \backslash x K[x]$ such that $f^{\circ N}=x^{s} P(x)^{n}$. Then there exist a positive integer $\tilde{s}$ and a non-constant polynomial $P_{1}(x) \in K[x] \backslash x K[x]$ such that $f^{\circ 4}=x^{\tilde{s}} P_{1}(x)^{n}$.

Proof. By Lemma 3.5, $f^{\circ 4}$ is neither cyclic nor dihedral. By applying Lemma 3.8 to the identity $f^{\circ 4} \circ f^{\circ(N-4)}=f^{\circ(N-4)} \circ f^{\circ 4}=x^{s} P(x)^{n}$, there exist linear polynomials $L_{3}, L_{4}$, positive integers $j, k$ each of which is coprime to $n$, and non-constant polynomials $P_{1}(x), P_{2}(x) \in K[x] \backslash x K[x]$ such that:

$$
f^{\circ 4}=x^{j} P_{1}(s)^{n} \circ L_{3}=L_{4}^{-1} \circ x^{k} P_{2}(x)^{n} .
$$

Since $f^{\circ 4}$ is neither cyclic nor dihedral, $x^{j} P_{1}(s)^{n}$ is neither cyclic nor dihedral. From $L_{4} \circ x^{j} P_{1}(x)^{n} \circ L_{3}=x^{k} P_{2}(x)^{n}$ and Lemma 3.9, we have that $L_{4}=\gamma x$ for some $\gamma \in K^{*}$. Hence $f^{\circ 4}=L_{4}^{-1} \circ x^{k} P_{2}(x)^{n}$ has the desired form.

The following result is well-known in Ritt's theory:

Lemma 3.11. Let $f \in K[x]$ be a disintegrated polynomial.

(a) If $g \in K[x]$ has degree at least 2 such that $g$ commutes with an iterate of $f$ then $g$ and $f$ have a common iterate.

(b) Let $M\left(f^{\infty}\right)$ denote the collection of all linear polynomials commuting with an iterate of $f$. Then $M\left(f^{\infty}\right)$ is a finite cyclic group under composition. 
(c) Let $\tilde{f} \in F[X]$ be a polynomial of lowest degree at least 2 such that $\tilde{f}$ commutes with an iterate of $f$. Then there exists $e>0$ relatively prime to the order of $M\left(f^{\infty}\right)$ such that $\tilde{f} \circ L=L^{\circ e} \circ \tilde{f}$ for every $L \in M\left(f^{\infty}\right)$.

(d) $\left\{\tilde{f}^{\circ m} \circ L: m \geq 0, L \in M\left(f^{\infty}\right)\right\}=\left\{L \circ \tilde{f}^{\circ m}: m \geq 0, L \in M\left(f^{\infty}\right)\right\}$, and these sets consist of exactly all the polynomials $g$ commuting with an iterate of $f$.

(e) The order of $M\left(f^{\infty}\right)$ is at most $\operatorname{deg}(\tilde{f}) / 2$.

Proof. For parts (a), (b), (c), and (d), see Proposition 2.3 and Remark 2.4 in [Ngu15]. For part (e), if $\tilde{f}$ is not cyclic, we have $M\left(f^{\infty}\right)=M\left(\tilde{f}^{\infty}\right)$ is a subgroup of $\Gamma(\tilde{f})$ which has at most $\operatorname{deg}(\tilde{f}) / 2$ elements by Lemma 3.6. If $\tilde{f}$ is cyclic, by taking a linear conjugation if necessary, we may assume that $\tilde{f}=x^{m}+c$ with $c \neq 0$. For every $n \geq 1, \tilde{f}^{\circ n}$ has the form $x^{m^{n}}+a x^{m^{n}-m}+\ldots$ for some $a \neq 0$. Hence $M\left(\tilde{f}^{\infty}\right)$ contains linear polynomials of the form $\zeta x$ where $\zeta$ is an $m$-th root of unity. Since $\tilde{f}(\zeta x)=\tilde{f}$, we conclude that $M\left(\tilde{f}^{\infty}\right)$ is trivial.

Lemma 3.12. Let $f \in K[x]$ be a disintegrated polynomial of degree $\delta$. Then there exists $n \leq \delta / 2$ such that the following hold. If $g$ is a non-constant polynomial commuting with an iterate of $f$ then $f^{\text {on }}$ commutes with $g$.

Proof. Let $\tilde{f}, M\left(f^{\infty}\right)$, and $e$ be as in the statement of Lemma 3.11. By part (d) of Lemma 3.11, it suffices to show that there is $n \leq \operatorname{deg}(\tilde{f}) / 2 \leq \delta / 2$ such that $\tilde{f}^{\circ}$ commutes with every element in $M\left(f^{\infty}\right)$. By part (c) of Lemma 3.11, $\tilde{f}^{\circ n} \circ L=L^{\circ e^{n}} \circ \tilde{f}^{\circ n}$ for every $L \in M\left(f^{\infty}\right)$. We simply choose $n$ such that $e^{n} \equiv 1$ modulo $\left|M\left(f^{\infty}\right)\right|$. Since $\left|M\left(f^{\infty}\right)\right| \leq \operatorname{deg}(\tilde{f}) / 2$, such an $n$ could be chosen to be at most $\operatorname{deg}(\tilde{f}) / 2$.

\section{Proof of Theorem 2.3 and Corollary 2.4}

We start with the following easy lemma:

Lemma 4.1. Let $A$ and $B$ be non-constant polynomials. Assume that $A \circ B$ is disintegrated, then $B \circ A$ is disintegrated.

Proof. Let $\alpha=\operatorname{deg}(A)$ and $\beta=\operatorname{deg}(B)$. Assume that $B \circ A$ is linearly conjugate to $x^{\alpha \beta}$, namely $B \circ A=L \circ x^{\alpha \beta} \circ L^{-1}$. By Lemma 3.1, there is a linear $L_{1}$ such that $B=L \circ x^{\beta} \circ L_{1}$, and $A=L_{1}^{-1} \circ x^{\alpha} \circ L^{-1}$. Then $A \circ B=L^{-1} \circ x^{\alpha \beta} \circ L$, contradiction. The case that $B \circ A$ is linearly conjugate to $T_{\alpha \beta}(x)$ is settled similarly.

Proof of Theorem 2.3. Assume there is a counter-example to Theorem 2.3. Among all such counter-examples, let $(f, g)$ satisfy the following properties:

(a) For every $N \leq 2 d^{4}$, there does not exist a polynomial that is semiconjugate to both $f^{\circ N}$ and $g^{\circ N}$. 
(b) There exist non-constant polynomials $p, q, \theta \in K[x]$ and $n \in \mathbb{N}$ (necessarily greater than $2 d^{4}$ ) such that $f^{\circ n} \circ p=p \circ \theta$ and $g^{\circ n} \circ q=$ $q \circ \theta$.

(c) The number $\operatorname{deg}(p)+\operatorname{deg}(q)$ is minimal among all counter-examples and all the quadruples $(\theta, p, q, n)$.

Write $\delta=\operatorname{deg}(f)=\operatorname{deg}(g)$. Let $D=\operatorname{gcd}(\delta, \operatorname{deg}(p))$.

Assume first that $D>1$. Then by Lemma 3.1, we can write $f=A \circ f_{0}$, $p=A \circ p_{0}$ with $\operatorname{deg}(A)=D$ and:

$$
f_{0} \circ f^{\circ(n-1)} \circ A \circ p_{0}=p_{0} \circ \theta .
$$

Hence $\left(f_{0} \circ A\right)^{\circ n} \circ p_{0}=p_{0} \circ \theta$. By Lemma 4.1, $f_{0} \circ A$ remains disintegrated. Because of $\operatorname{deg}\left(p_{0}\right)<\operatorname{deg}(p)$ and the minimality of $\operatorname{deg}(p)+\operatorname{deg}(q)$, the pair $\left(f_{0} \circ A, g\right)$ is not a counter-example to Theorem 2.3. So there is $N \leq 2 d^{4}$ such that there exists a common polynomial $B$ that is semiconjugate to both $\left(f_{0} \circ A\right)^{\circ N}$ and $g^{\circ N}$. This implies that $B$ is semiconjugate to both $f^{\circ N}=\left(A \circ f_{0}\right)^{\circ N}$ and $g^{\circ N}$, contradiction. We conclude that $D=1$.

So, from now on, we may and do assume that $\operatorname{gcd}(\delta, \operatorname{deg}(p))=1$. Similarly, $\operatorname{gcd}(\delta, \operatorname{deg}(q))=1$.

Let $\eta=\theta^{\circ 4}$, it will be more convenient to work with the following identities:

$$
f^{\circ 4 n} \circ p=p \circ \eta ; g^{\circ 4 n} \circ q=q \circ \eta
$$

Write $b=\operatorname{deg}(p)$ and $\tilde{b}=\operatorname{deg}(q)$. By Lemma 3.2, there exist positive integers $c \equiv \delta^{4 n}$ modulo $b$ and $\tilde{c} \equiv \delta^{4 n}$ modulo $\tilde{b}$ together with linear polynomials $\ell_{1}, \ell_{2}, \ell_{3}, \ell_{4}$ and non-constant polynomials $P$ and $Q$ such that the following groups of identities hold:

(i) $\ell_{1} \circ f^{\circ 4 n} \circ \ell_{1}^{-1}=x^{c} P(x)^{b}, \ell_{1} \circ p \circ \ell_{2}^{-1}=x^{b}$, and $\ell_{2} \circ \eta \circ \ell_{2}^{-1}=x^{c} P\left(x^{b}\right)$.

(ii) $\ell_{3} \circ g^{\circ 4 n} \circ \ell_{3}^{-1}=x^{\tilde{c}} Q(x)^{\tilde{b}}, \ell_{3} \circ q \circ \ell_{4}^{-1}=x^{\tilde{b}}$, and $\ell_{4} \circ \eta \circ \ell_{4}^{-1}=x^{\tilde{c}} Q\left(x^{\tilde{b}}\right)$. We may assume that $P(0) \neq 0$ and $Q(0) \neq 0$ (since $f$ and $g$ are not linearly conjugate to the monomial $\left.x^{\delta}\right)$. Write $f_{1}:=\ell_{1} \circ f^{\circ 4} \circ \ell_{1}^{-1}, p_{1}:=\ell_{1} \circ p \circ \ell_{2}^{-1}=$ $x^{b}, g_{1}:=\ell_{3} \circ g^{\circ 4} \circ \ell_{3}^{-1}$, and $q_{1}:=\ell_{3} \circ q \circ \ell_{4}^{-1}=x^{\tilde{b}}$. We now consider three cases.

Case 1: $b=\tilde{b}=1$. Then we have that $f^{\circ 4 n}$ and $g^{\circ 4 n}$ are both linearly conjugate to $\eta$. Hence there is a linear $L$ such that $f^{\circ 4 n}=\left(L \circ g^{4} \circ L^{-1}\right)^{\circ n}$. By Lemma 3.5 and Lemma 3.7, there is $N \leq \frac{d^{4}}{2}$ such that $f^{\circ 4 N}$ is linearly conjugate to $g^{\circ 4 N}$. Hence $g^{\circ 4 N}$ is semiconjugate to both $f^{\circ 4 N}$ and $g^{\circ 4 N}$, contradicting the assumption that $(f, g)$ is a counter-example.

Case 2: $b \geq 2$ and $\tilde{b}=1$. Then Lemma 3.10 gives that $f_{1}=x^{j} P_{1}(x)^{b}$ for some non-constant $P_{1}(x) \in \mathbb{C}[x] \backslash x \mathbb{C}[x]$ and positive integer $j$ coprime to $b$. Write $W(x)=x^{j} P_{1}\left(x^{b}\right)$ (which is not cyclic by part (e) of Lemma 3.6), we have:

$$
f_{1} \circ p_{1}=p_{1} \circ W
$$

This implies:

$$
f_{1}^{\circ n} \circ p_{1}=p_{1} \circ W^{\circ n}
$$


Since $f_{1}^{\circ n} \circ p_{1}=p_{1} \circ\left(\ell_{2} \circ \eta \circ \ell_{2}^{-1}\right)$, we have that $W^{\circ n}=\zeta\left(\ell_{2} \circ \eta \circ \ell_{2}^{-1}\right)$ for some $b$-th root of unity $\zeta$. Since $\tilde{b}=1, g^{\circ 4 n}$ is linearly conjugate to $\eta$. Hence there is a linear $\ell$ such that:

$$
W^{\circ n}=\zeta\left(\ell \circ g^{\circ 4 n} \circ \ell^{-1}\right) .
$$

By Lemma 3.7, there is $N \leq \frac{d^{4}}{2}$ such that $W^{\circ N}$ is linearly conjugate to $g^{\circ 4 N}$. Since $W^{\circ N}$ is semiconjugate to $f_{1}^{\circ N}$, so is $g^{\circ 4 N}$. Since $f^{\circ 4 N}$ is linearly conjugate to $f_{1}^{\circ N}$, we have that $g^{\circ 4 N}$ is semiconjugate to $f^{\circ 4 N}$. Hence $g^{\circ 4 N}$ is semiconjugate to both $g^{\circ 4 N}$ and $f^{\circ 4 N}$, contradicting the assumption that $(f, g)$ is a counter-example.

Case 3: $b \geq 2$ and $\tilde{b} \geq 2$. By using similar arguments as in Case 2, we have that $f_{1}=x^{j} P_{1}(x)^{b}$ and $g_{1}=x^{k} Q_{1}(x)^{\tilde{b}}$ for some non-constant $P_{1}, Q_{1} \in$ $K[x] \backslash x K[x]$ and positive integers $j, k$ satisfying $\operatorname{gcd}(j, b)=\operatorname{gcd}(k, \tilde{b})=1$. Write $W:=x^{j} P_{1}\left(x^{b}\right)$ and $V:=x^{k} Q_{1}\left(x^{\tilde{b}}\right)$ (which are not cyclic by part (e) of Lemma 3.6), we have:

$$
f_{1} \circ p_{1}=p_{1} \circ W \text { and } g_{1} \circ q_{1}=q_{1} \circ V \text {. }
$$

From $f_{1}^{\circ n} \circ p_{1}=p_{1} \circ\left(\ell_{2} \circ \eta \circ \ell_{2}^{-1}\right)$ and $g_{1}^{\circ n} \circ q_{1}=q_{1} \circ\left(\ell_{4} \circ \eta \circ \ell_{4}^{-1}\right)$, we have:

$$
W^{\circ n}=\zeta\left(\ell_{2} \circ \eta \circ \ell_{2}^{-1}\right) \text { and } V^{\circ n}=\mu\left(\ell_{4} \circ \eta \circ \ell_{4}^{-1}\right)
$$

for some $b$-th root of unity $\zeta$ and $\tilde{b}$-th root of unity $\mu$. Hence there are linear polynomials $L$ and $L_{1}$ such that:

$$
W^{\circ n}=L \circ\left(L_{1} \circ V^{\circ n} \circ L_{1}^{-1}\right) .
$$

By Lemma 3.7, there exists $N \leq \frac{d^{4}}{2}$ such that $W^{\circ N}$ is linearly conjugate to $V^{\circ N}$. Since $W^{\circ N}$ (respectively $V^{\circ N}$ ) is semiconjugate to $f_{1}^{\circ N}$ (respectively $g_{1}^{\circ N}$ ), and $f_{1}^{\circ N}$ (respectively $g_{1}^{\circ N}$ ) is linearly conjugate to $f^{\circ 4 N}$ (respectively $g^{\circ 4 N}$ ), we conclude that there is a common polynomial semiconjugate to both $f^{\circ 4 N}$ and $g^{\circ 4 N}$. This contradicts the assumption that $(f, g)$ is a counterexample.

Proof of Corollary 2.4. By Theorem 2.3, we define $c_{1}(d, 2)=2 d^{4}$ for every integer $d \geq 2$. Let $n \geq 3$, and assume that we have determined $c_{1}(d, n)$ for all smaller values of $n$ and all $d \geq 2$. By the induction hypothesis, there exist positive integers $N_{1} \leq c_{1}(d, n-1)$ and $\theta_{1} \in K[x]$ such that $\theta_{1}$ is semiconjugate to $f_{i}^{\circ N_{1}}$ for $1 \leq i \leq n-1$.

We have $\theta_{1} \approx f_{1}^{\circ N_{1}} \approx f_{n}^{\circ N_{1}}$. By Theorem 2.3, there exist $N_{2} \leq 2 d^{4 N_{1}}$ and a polynomial $\theta$ that is semiconjugate to both $\theta_{1}^{\circ N_{2}}$ and $f_{n}^{\circ N_{1} N_{2}}$. We have that $\theta$ is semiconjugate to $f_{i}^{\circ N_{1} N_{2}}$ for every $1 \leq i \leq n$. Define $c_{1}(d, n)=$ $c_{1}(d, n-1) 2 d^{4 c_{1}(d, n-1)}$ so that $N:=N_{1} N_{2} \leq c_{1}(d, n)$; this finishes the proof of Corollary 2.4. 


\section{The Medvedev-Scanlon Classification of Periodic SUBVARIETIES}

Let $n \in \mathbb{N}$. The main results of the paper [MS14] provide a description of periodic subvarieties of $\mathbb{A}_{K}^{n}$ under coordinate-wise self-maps of the form

$$
\left(x_{1}, \ldots, x_{n}\right) \mapsto\left(f_{1}\left(x_{1}\right), \ldots, f_{n}\left(x_{n}\right)\right)
$$

where $f_{1}, \ldots, f_{n} \in K[x]$ are non-constant. The main reference is the original paper by Medvedev-Scanlon [MS14] together with further remarks in [Ngu15] and [GN]. We start with the following:

Proposition 5.1. Let $L_{1}, \ldots, L_{r} \in K[x]$ be linear polynomials, let $M_{1}, \ldots, M_{s} \in$ $K[x]$ be polynomials of degree at least 2 such that each $M_{i}$ is linearly conjugate to $x^{\operatorname{deg}\left(M_{i}\right)}$ or $\pm T_{\operatorname{deg}\left(M_{i}\right)}(x)$ for $1 \leq i \leq s$, and let $f_{1}, \ldots, f_{t} \in K[x]$ be disintegrated polynomials. Write $n=r+s+t$ and let $\phi$ be the coordinatewise self-map of $\mathbb{A}^{n}$ induced by the polynomials $L_{i}$ 's, $M_{i}$ 's, and $f_{i}$ 's. Then every irreducible $\phi$-periodic subvariety $V$ has the form $V_{1} \times V_{2} \times V_{3}$ where $V_{1}, V_{2}$, and $V_{3}$ respectively are periodic under the coordiniate-wise self-maps $L_{1} \times \cdots \times L_{r}, M_{1} \times \cdots \times M_{s}$, and $f_{1} \times \cdots \times f_{t}$.

Proof. This is a consequence of [MS14, Theorem 2.30].

We now study periodic subvarieties under the coordinate-wise self-map $f_{1} \times \cdots \times f_{n}$ where each $f_{i} \in K[x]$ is disintegrated:

Proposition 5.2. Let $n \geq 2$, let $f_{1}, \ldots, f_{n} \in K[x]$ be disintegrated polynomials, and let $\phi=f_{1} \times \cdots \times f_{n}$ be the induced coordinate-wise self-map of $\mathbb{A}_{K}^{n}$. Then every irreducible $\phi$-periodic subvariety $V$ of $\mathbb{A}_{K}^{n}$ is an irreducible component of

$$
\bigcap_{1 \leq i<j \leq n} \pi_{i j}^{-1}\left(\pi_{i j}(Z)\right)
$$

where $\pi_{i j}$ denotes the projection from $\mathbb{A}_{K}^{n}$ to the $(i, j)$-factor $\mathbb{A}_{K}^{2}$.

Proof. See Proposition 2.21 and Fact 2.25 in [MS14].

Recall the equivalence relation $\approx$ in Definition 2.2. Note that $f \approx g$ if and only if the coordinate-wise self-map $f \times g$ of $\mathbb{A}^{2}$ admits an irreducible periodic curve whose projection to each factor $\mathbb{A}^{1}$ is non-constant (see [MS14, Corollary 2.35]). Proposition 5.2 implies the following:

Proposition 5.3. Let $s, n_{1}, \ldots, n_{s}$ be positive integers. For $1 \leq i \leq s$ and $1 \leq j \leq n_{i}$, let $f_{i, j} \in K[x]$ be a disintegrated polynomial such that the $f_{i, j}$ 's form exactly s equivalence classes each of which being $\left(f_{i, j}\right)_{1 \leq j \leq n_{i}}$ for $1 \leq i \leq s$. For $1 \leq i \leq s$, let $\phi_{i}=f_{i, 1} \times \cdots \times f_{i, n_{i}}$ be the induced coordinatewise self-map of $\mathbb{A}^{n_{i}}$. Write $n=n_{1}+\ldots+n_{s}$, identify $\mathbb{A}^{n}=\mathbb{A}^{n_{1}} \times \cdots \times \mathbb{A}^{n_{s}}$, and let $\phi=\phi_{1} \times \cdots \times \phi_{s}$ be the induced coordinate-wise self-map of $\mathbb{A}^{n}$. The following hold:

(i) Every irreducible $\phi$-periodic subvariety $V$ of $\mathbb{A}^{n}$ has the form $V_{1} \times$ $\cdots \times V_{s}$ where each $V_{i}$ is a $\phi_{i}$-periodic subvariety of $\mathbb{A}^{n_{i}}$ for $1 \leq i \leq s$. 
(ii) Let $1 \leq i \leq s$. There exists $N_{i} \in \mathbb{N}$, non-constant polynomials $\theta_{i}$ and $p_{i, j}$ for $1 \leq j \leq n_{i}$ such that $f_{i, j}^{\circ N_{i}} \circ p_{i, j}=p_{i, j} \circ \theta_{i}$. In other words, we have the commutative diagram (with $p_{i}:=p_{i, 1} \times \cdots \times p_{i, n_{i}}$ ):

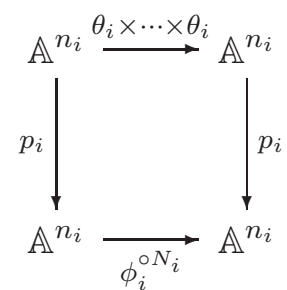

Proof. Part (a) is a consequence of [MS14, Theorem 2.30]. Part (b) (which was alluded in Section 2) is proved in [GN, Section 7.1].

Remark 5.4. We note that (according to part (ii) of Proposition 5.3), if there exists a periodic $\phi$-subvariety $V$ as in Proposition 5.3, then $\operatorname{deg}\left(f_{i, 1}\right)=\cdots=$ $\operatorname{deg}\left(f_{i, n_{i}}\right)$ for each $i$.

Proposition 5.3 shows that it suffices to treat self-maps of $\mathbb{A}^{n}$ of the form $f \times \cdots \times f$ of where $f$ is disintegrated. Let $x_{1}, \ldots, x_{n}$ denote the coordinate functions of each factor $\mathbb{A}_{K}^{1}$ of $\mathbb{A}_{K}^{n}$. We have:

Proposition 5.5. Let $n \in \mathbb{N}$ and $f \in K[x]$ be disintegrated. Let $\phi:=f \times$ $\cdots \times f$ be the induced coordinate-wise self-map of $\mathbb{A}_{K}^{n}$. Then every irreducible $\phi$-periodic subvariety $V$ of $\mathbb{A}_{K}^{n}$ is defined by a collection of equations of the following forms:

(i) $x_{i}=\zeta$ for some $1 \leq i \leq n$ and some $\zeta \in K$ that is $f$-periodic.

(ii) $x_{i}=g\left(x_{j}\right)$ for some $1 \leq i \neq j \leq n$ and some non-constant $g \in K[x]$ that commutes with an iterate of $f$.

Proof. See [MS14, Theorem 6.24].

\section{Proof of Theorem 1.1}

Obviously, we can define $c(d, 1)=1$ for every $d \geq 2$ since $\mathbb{A}^{1}$ is the only periodic subvariety satisfying the condition of the theorem when $n=1$. Let $n \geq 2$, assume that $c(d, n)$ has been defined for all smaller values of $n$ and for all $d \geq 2$. Recall the constant $c_{1}(d, n)$ in Theorem 2.4, define:

$$
c(d, n):=\max \left\{c(d, n-1)^{n-1}, \frac{d^{c_{1}(d, n)}}{2}\right\} .
$$

Under the equivalence relation $\approx$, assume that $f_{1}, \ldots, f_{n}$ belong to exactly $s$ equivalence classes whose sizes are $n_{1}, \ldots, n_{s}$. We consider two cases:

Case 1: $s \geq 2$. By Proposition 5.3, after rearranging the factors $\mathbb{A}^{1}$ of $\mathbb{A}^{n}$, every irreducible $\varphi$-periodic subvariety $V$ of $\mathbb{A}^{n}$ has the form $V_{1} \times \cdots \times V_{s}$. By the induction hypothesis, the period of each $V_{i}$ is at most $c\left(d, n_{i}\right)$. Hence the period of $V$ is at most:

$$
c\left(d, n_{1}\right) \ldots c\left(d, n_{s}\right) \leq c(d, n-1)^{n-1} \leq c(d, n) .
$$


Case 2: $s=1$. By Theorem 2.4, there exist a positive integer $N \leq$ $c_{1}(d, n)$ and non-constant polynomials $\theta, p_{1}, \ldots, p_{n} \in K[x]$ such that $f_{i}^{\circ N_{\circ}}$ $p_{i}=p_{i} \circ \theta$ for $1 \leq i \leq n$. Let $\phi:=\theta \times \cdots \times \theta$ and $\rho:=p_{1} \times \cdots \times p_{n}$ be the induced coordinate-wise self-maps of $\mathbb{A}^{n}$. We have the commutative diagram:

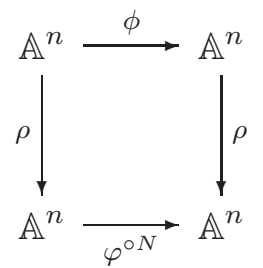

Since $V$ is periodic under $\varphi^{\circ N}$, some irreducible component $\tilde{V}$ of $\rho^{-1}(V)$ is periodic under $\phi$. Since the projection from $V$ to each factor $\mathbb{A}^{1}$ is nonconstant, so is the projection from $\tilde{V}$ to each factor $\mathbb{A}^{1}$. Therefore, by Proposition 5.5, $\tilde{V}$ is defined by equations of the form $x_{i}=g\left(x_{j}\right)$ for some $1 \leq i \leq j$ and some non-constant $g \in K[x]$ commuting with an iterate of $\theta$. By Lemma 3.12, there is $N_{1} \leq \frac{\operatorname{deg}(\theta)}{2}=\frac{d^{N}}{2}$ such that $\theta^{\circ N_{1}}$ commutes with $g$. Hence the $\phi$-period of $\tilde{V}$ is at most $N_{1}$. Consequently, the $\varphi$-period of $V$ is at most:

$$
N_{1} \leq \frac{d^{N}}{2} \leq \frac{d^{c_{1}(d, n)}}{2} \leq c(d, n)
$$

This finishes the proof of Theorem 1.1.

\section{Proof of Theorem 1.2}

7.1. A simple reduction. Obviously, it suffices to prove Theorem 1.2 when $C$ is irreducible. As shown in the proof of [BGKT12, Theorem 1.4] (see also the proof of Conjecture 5.10.0.17 as a consequence of Conjecture 5.10.0.18 in $[\mathrm{BGT}])$, it suffices to prove Theorem 1.2 when $m=2$. Also, the theorem (in the case $m=2$ ) follows immediately if the curve $C$ does not project dominantly onto both axes of $\mathbb{A}^{2}$; hence, in particular, we may assume that the polynomials $F_{i}$ 's are not constant. In addition, write $\alpha:=\left(\alpha_{1}, \alpha_{2}\right)$ and if $\alpha_{i}$ is preperiodic under the action of $F_{i}$ for some $i \in\{1,2\}$, then the result also follows easily (see [BGT, Proposition 3.1.2.9]). Furthermore, if $C$ is periodic under the action of $\Phi$, then Theorem 1.2 follows readily. Therefore, all we need to prove is the following theorem.

Theorem 7.1. Let $F_{1}(x), F_{2}(x) \in K[x]$ be non-constant and let $\alpha_{1}, \alpha_{2} \in K$ such that for each $i=1,2$, we have that $\alpha_{i}$ is not $F_{i}$-preperiodic. Let $\alpha:=\left(\alpha_{1}, \alpha_{2}\right)$, let $\Phi: \mathbb{A}_{K}^{2} \longrightarrow \mathbb{A}_{K}^{2}$ be the endomorphism given by $\Phi(x, y):=$ $\left(F_{1}(x), F_{2}(y)\right)$, and let $C \subset \mathbb{A}_{K}^{2}$ be an irreducible curve which is not $\Phi$ periodic. Assume that the projection from $C$ to each factor $\mathbb{A}_{K}^{1}$ is not constant. Then the set $S:=\left\{n \in \mathbb{N}: \Phi^{n}(\alpha) \in C\right\}$ is finite.

The rest of this section is dedicated to the proof of Theorem 7.1. Note that Theorem 7.1 in the case $K=\overline{\mathbb{Q}}$ follows from recent work of Xie [Xie]. 
In the next subsection, we explain how to combine Theorem 1.1 together with standard specialization arguments to settle the case of general $K$.

7.2. Proof of Theorem 7.1. We work under the hypotheses of Theorem 7.1.

Let $\mathcal{K} \subset K$ be a finitely generated field over $\overline{\mathbb{Q}}$ such that $\alpha_{1}, \alpha_{2} \in \mathcal{K}$, the coefficients of $F_{1}$ and of $F_{2}$ are in $\mathcal{K}$, and also the curve $C$ is defined by $G(x, y)=0$ for $G(x, y) \in \mathcal{K}[x, y]$. We argue by induction on the transcendence degree $e$ of $\mathcal{K} / \overline{\mathbb{Q}}$. The case $e=0$ has been obtained by Xie [Xie, Theorem 0.3]. So, we may assume $\mathcal{K}$ is a function field of transcendence degree 1 over some subfield $E$, and moreover, we may assume that Theorem 7.1 holds if $F_{1}, F_{2}, \alpha_{1}, \alpha_{2}$ and $C$ are all defined over $\bar{E}$. If both $F_{1}$ and $F_{2}$ are linear polynomials, then $\Phi$ is an automorphism of $\mathbb{A}^{2}$ and in this case, the Dynamical Mordell-Lang Conjecture is known to hold as proven by Bell [Bel06]. So, from now on, assume that $\operatorname{deg}\left(F_{2}\right) \geq 2$. Assume that the set $\left\{m \in \mathbb{N}: \Phi^{\circ m}(\alpha) \in C\right\}$ is infinite and we will arrive at a contradiction.

At the expense of replacing $E$ by its algebraic closure inside $\mathcal{K}$, we may assume that $\mathcal{K}$ is the function field of a smooth geometrically irreducible projective curve $X$ defined over $E$. Then for all but finitely many point $\mathfrak{p}$ of $X(\bar{E})$ we can specialize both $F_{1}$ and $F_{2}$ at those points and the corresponding specialization $F_{1, \mathfrak{p}}, F_{2, \mathfrak{p}} \in \bar{E}[x]$ satisfy the following properties:

(i) $\operatorname{deg}\left(F_{i, \mathfrak{p}}\right)=\operatorname{deg}\left(F_{i}\right)$ for each $i=1,2$;

(ii) for $i=1,2$, if $F_{i}$ is disintegrated, then $F_{i, \mathfrak{p}}$ is disintegrated.

Condition (i) is verified by all places of good reduction for the polynomials $F_{1}$ and $F_{2}$. Condition (ii) is also satisfied by all but finitely many places in $X(\bar{E})$ according to [BGKT12, Proposition 7.8]. In addition to conditions (i)-(ii), the specialization at all but finitely many points of $X(\bar{E})$ satisfies the following properties:

(iii) each coefficient of $G$ is integral at $\mathfrak{p}$; and

(iv) the curve $C_{\mathfrak{p}}$ which is the zero locus of $G_{\mathfrak{p}}(X, Y)=0$ (where $G_{\mathfrak{p}}$ is the polynomial obtained by reducing each coefficient of $G$ modulo $\mathfrak{p}$ ) is also geometrically irreducible (over $\bar{E}$ ). In addition, the projection from $C_{\mathfrak{p}}$ to each factor $\mathbb{A}_{\bar{E}}^{1}$ is not constant.

Clearly, condition (iii) is satisfied by all but finitely many places in $X(\bar{E})$. The same is true regarding the first assertion of condition (iv) (this follows from the Bertini-Noether theorem, see [FJ08, pp. 170]). Since $\operatorname{deg}_{y}(G)=$ $\operatorname{deg}_{y}\left(G_{\mathfrak{p}}\right)$ and $\operatorname{deg}_{x}(G)=\operatorname{deg}_{x}\left(G_{\mathfrak{p}}\right)$ for all but finitely many $\mathfrak{p}$, the second assertion in condition (iv) also holds. Finally, according to [GTZ08, Proposition 6.2 ], there are infinitely many $\mathfrak{p} \in X(\bar{E})$ such that:

(v) the reduction $\alpha_{2, \mathfrak{p}}$ of $\alpha_{2}$ modulo $\mathfrak{p}$ is not preperiodic under $F_{2, \mathfrak{p}}$.

We denote by $T$ the infinite set of places $\mathfrak{p}$ satisfying conditions (i)-(v). Then for each such $\mathfrak{p} \in T$, the orbit of $\left(\alpha_{1, \mathfrak{p}}, \alpha_{2, \mathfrak{p}}\right)$ under $F_{1, \mathfrak{p}} \times F_{2, \mathfrak{p}}$ intersects the curve $C_{\mathfrak{p}}$ in infinitely many points. So, by the inductive hypothesis, we know that $C_{\mathfrak{p}}$ is periodic under $F_{1, \mathfrak{p}} \times F_{2, \mathfrak{p}}$. Since the projection from $C_{\mathfrak{p}}$ to each 
$\mathbb{A}_{\bar{E}}^{1}$ is non-constant and $\operatorname{deg}\left(F_{2}\right) \geq 2$, Proposition 5.1 and Proposition 5.3 show that it suffices to consider the following two cases.

Case 1. $F_{1}$ and $F_{2}$ are disintegrated polynomials of the same degree $\delta$ (see Remark 5.4 applied to the polynomials $F_{1, \mathfrak{p}}$ and $F_{2, \mathfrak{p}}$, and also note that $\operatorname{deg}\left(F_{1}\right)=\operatorname{deg}\left(F_{1, \mathfrak{p}}\right)$ and $\operatorname{deg}\left(F_{2}\right)=\operatorname{deg}\left(F_{2, \mathfrak{p}}\right)$ for $\left.\mathfrak{p} \in T\right)$. By Theorem 1.1, there is a constant $N$ depending only on $\delta$ such that $\left(F_{1, \mathfrak{p}} \times F_{2, \mathfrak{p}}\right)^{\circ N}\left(C_{\mathfrak{p}}\right)=C_{\mathfrak{p}}$ for every $\mathfrak{p} \in T$. Since $T$ is infinite, we have $\Phi^{\circ N}(C)=C$. This gives that $C$ is $\Phi$-periodic, contradiction.

Case 2. Both $F_{1}$ and $F_{2}$ are not disintegrated. Write $\delta_{i}=\operatorname{deg}\left(F_{i}\right)$ for $i=1,2$. It is more convenient to regard $\Phi$ as a self-map of $\left(\mathbb{P}^{1}\right)^{2}$ and replace $C$ by its Zariski closure in $\left(\mathbb{P}^{1}\right)^{2}$. For $i=1,2$, define $\nu_{i}(x)=x$ if $F_{i}$ is linearly conjugate to a monomial and $\nu_{i}(x)=x+\frac{1}{x}$ if $F_{i}$ is linearly conjugate to $\pm T_{\delta_{i}}(x)$. Let $\nu=\nu_{1} \times \nu_{2}$ be the corresponding coordinate-wise self-map of $\left(\mathbb{P}^{1}\right)^{2}$. We have the commutative diagram:

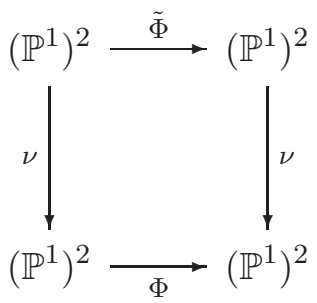

where $\tilde{\Phi}$ has the form $(x, y) \mapsto\left( \pm x^{\delta_{1}}, \pm x^{\delta_{2}}\right)$. Pick $\beta \in \nu^{-1}(\alpha)$, then there is an irreducible component $\tilde{C}$ of $\nu^{-1}(C)$ having an infinite intersection with the $\tilde{\Phi}$-orbit of $\beta$. In this case, it is well-known that $\tilde{C}$ is $\tilde{\Phi}$-periodic (see either [GT09, Theorem 1.8], or [BGT10, Theorem 1.3] with the remark that $\tilde{\Phi}$ induces an étale endomorphism of $\mathbb{G}_{\mathrm{m}}^{2}$ ). Therefore $C$ is $\Phi$-periodic, contradiction. This concludes the proof of Theorem 7.1 and therefore the proof of Theorem 1.2.

\section{REFERENCES}

[Bel06] J. P. Bell, A generalized Skolem-Mahler-Lech theorem for affine varieries, J. Lond. Math. Soc.(2) 73 (2006), 367-379.

[BG06] E. Bombieri and W. Gubler, Heights in Diophantine geometry, New Mathematical Monographs, vol. 4, Cambridge University Press, Cambridge, 2006.

[BGKT12] R. Benedetto, D. Ghioca, P. Kurlberg, and T. Tucker, A case of the dynamical Mordell-Lang conjecture with an appendix by U. Zannier, Math. Ann. 352 (2012), 1-26.

[BGT] J. Bell, G. Ghioca, and T. Tucker, The Dynamical Mordell-Lang Conjecture, to appear in the AMS Mathematical Surveys and Monographs series, 300 pages, 2015 .

[BGT10] - The dynamical Mordell-Lang problem for étale maps, Amer. J. Math. 132 (2010), 1655-1675.

[Eng41] H. T. Engstrom, Polynomial substituitions, Amer. J. Math. 63 (1941), 249255.

[FJ08] M. D. Fried and M. Jarden, Field arithmetic, third ed., A series of modern surveys in mathematics, vol. 11, Springer, 2008. 
[GN] D. Ghioca and K. D. Nguyen, Dynamical anomalous subvarieties: structure and bounded height theorems, arXiv:1408.5455, 2014.

[GT09] D. Ghioca and T. Tucker, Periodic points, linearizing maps, and the dynamical Mordell-Lang problem, J. Number Theory (2009), no. 129, 1392-1403.

[GTZ08] D. Ghioca, T. Tucker, and M. Zieve, Intersections of polynomial orbits, and a dynamical Mordell-Lang conjecture, Invent. Math. 171 (2008), 463-483.

[GTZ12] Linear relations between polynomial orbits, Duke Math. J. 161 (2012), 1379-1410.

[Ino11] H. Inou, Extending local analytic conjugacies, Trans. Amer. Math. Soc. 363 (2011), 331-343.

[MS14] A. Medvedev and T. Scanlon, Invariant varieties for polynomial dynamical systems, Ann. of Math. (2) 179 (2014), 81-177.

[Ngu15] K. Nguyen, Some arithmetic dynamics of diagonally split polynomial maps, Int. Math. Res. Not. IMRN 2015 (2015), 1159-1199.

[Pak] F. Pakovich, Polynomial semiconjugacies, decompositions of iterations, and invariant curves, arXiv:1505.06351, 2015

[Rit22] J. F. Ritt, Prime and composite polynomials, Trans. Amer. Math. Soc. 23 (1922), 51-66.

[Sch00] A. Schinzel, Polynomials with special regard to reducibility, Encyclopedia of mathematics and its applications, vol. 77, Cambridge University Press, 2000.

[Tor88] P. Tortrat, Sur la composition des polynômes, Colloq. Math. 55 (1988), 329353.

[Xie] J. Xie, The Dynamical Mordell-Lang conjecture for polynomial endomorphisms of the affine plane, arXiv:1503.00773, 2015.

[Zan93] U. Zannier, Ritt's second theorem in arbitrary characteristic, J. Reine Angew. Math. 445 (1993), 175-203.

[ZM] M. E. Zieve and P. Müller, On Ritt's polynomial decomposition theorems, arXiv:0807.3578, 2008.

Dragos Ghioca, Department of Mathematics, University of British Columbia, VANCOUVER, BC V6T 1Z2, CANADA

E-mail address: dghioca@math.ubc.ca

Khoa D. Nguyen, Department of Mathematics, University of British Columbia, And Pacific Institute for The Mathematical Sciences, Vancouver, BC V6T 1Z2, CANADA

E-mail address: dknguyen@math.ubc.ca

$U R L$ : www.math.ubc.ca/ ${ }^{\sim}$ knguyen 